\title{
THE SCHUBERT CALCULUS, BRAID RELATIONS, AND GENERALIZED COHOMOLOGY
}

\author{
PAUL BRESSLER AND SAM EVENS
}

\begin{abstract}
Let $X$ be the flag variety of a compact Lie group and let $h^{*}$ be a complex-oriented generalized cohomology theory. We introduce operators on $h^{*}(X)$ which generalize operators introduced by Bernstein, Gel' fand, and Gel'fand for rational cohomology and by Demazure for $K$-theory. Using the Becker-Gottlieb transfer, we give a formula for these operators, which enables us to prove that they satisfy braid relations only for the two classical cases, thereby giving a topological interpretation of a theorem proved by the authors and extended by Gutkin.
\end{abstract}

One of the central issues in Lie theory is the geometry of the flag variety associated to a compact Lie group $G$. An important problem concerning the flag variety is the Schubert calculus, which studies the ring structure of the cohomology of the flag variety. Work initiated by Borel, Bott and Kostant, which culminated in a paper by Bernstein, Gel'fand and Gel'fand [BGG], gave a complete solution to the problem. Demazure studied the same problem for $K$-theory. Moreover, these techniques have been generalized to the Kač-Moody situation by Kač-Peterson, Kostant-Kumar, and others. This work has focussed on algebro-geometric properties of the flag variety.

Here, on the other hand we study the flag variety from the point of view of algebraic topology. As a consequence, not only do we recover the classical results described above, but we extend these results to a certain class of cohomology theories-those which are complex-oriented. Examples of complex-oriented theories include ordinary cohomology, $K$-theory, complex cobordism, and elliptic cohomology. Since the context we have chosen in very general, the proofs are universal and are often simpler than the classical arguments.

In the work of BGG, a crucial role is played by operators $A_{i}$ associated to each simple reflection $s_{i}$ of the Weyl group of $G$ (defined by Demazure in $K$-theory). These operators $A_{i}$ satisfy the braid relations, which are the relations between pairs of simple reflections. In this paper, we generalize the $A_{i}$ to give operators $D_{i}$ acting on $h^{*}(G / T)$ for any complex-oriented theory $h^{*}$. We prove that braid relations are satisfied only for cohomology theories with the formal group law of rational cohomology or of $K$-theory (Theorem

Received by the editors June 21, 1988.

1980 Mathematics Subject Classification (1985 Revision). Primary 55N20, 57T15.

The second author was supported by an NSF graduate fellowship. 
3.7). In particular, the braid relations are not satisfied in the cases of complex cobordism and elliptic cohomology. This result relies on a previous result of ours, and its generalization by Gutkin, and answers a question posed by Kostant.

The main point of the argument is that one can give a formula for the operators by using a formula of Brumfiel and Madsen for the Becker-Gottlieb transfer. This computation is explained in $\S 1$. In $\S 2$, this formula is applied to ordinary cohomology and to $K$-theory. In $K$-theory, it gives an easy derivation of the Weyl character formula. In ordinary cohomology, we obtain a new proof of some results of Akyildiz and Carrell [2]. $\S 3$ is concerned with braid relations. In a later paper we will give a geometric interpretation of Theorem 3.7 and use this interpretation to give a complete description of the ring structure of $h^{*}(G / T)$ for complex-oriented theories $h^{*}$, generalizing the work of BGG and Demazure.

The authors would like to thank Haynes Miller for continuous help and encouragement. We would also like to thank Bert Kostant for suggesting the problem, and David Blanc and Hal Sadofsky for some helpful conversations. In addition, we would like to thank Peter Landweber for some suggestions.

\section{TOPOLOGICAL PRELIMINARIES}

In this section we discuss the Becker-Gottlieb transfer and the Gysin map for complex-oriented cohomology theories. Let $h^{*}$ be a generalized cohomology theory, so $h^{*}$ is a functor from topological spaces to graded abelian groups satisfying all of the Eilenberg-Steenrod axioms except the dimension axiom. Suppose in addition that $h^{*}$ is multiplicative, so that $h^{*}(X)$ has a ring structure.

Let $\pi: E \rightarrow B$ be a fiber bundle associated to a principal bundle, and assume the fiber $F$ is a compact manifold. The Becker-Gottlieb transfer is a stable map from $B$ to $E$. It induces the transfer homomorphism

$$
\tau(\pi)^{*}: h^{i}(E) \rightarrow h^{i}(B)
$$

This is not a ring homomorphism, but is an $h^{*}(B)$-module homomorphism, so

$$
\tau(\pi)^{*}\left(x \cdot \pi^{*}(y)\right)=\tau(\pi)^{*}(x) \cdot y .
$$

The Becker-Gottlieb transfer generalizes the notion of transfer for a finite covering.

A cohomology theory $h^{*}$ is said to be complex-oriented if it satisfies the following property. Let $\tilde{h}^{*}$ denote reduced cohomology and let $i: \mathbf{C P}(1) \hookrightarrow$ $\mathbf{C P}(\infty)$ be the canonical inclusion of $\mathbf{C P}(1)=S^{2}$ into infinite complex projective space.

Definition 1.1. $h^{*}$ is a complex-orientable cohomology theory if there exists an element $x \in \tilde{h}^{*}(\mathbf{C P}(\infty))$ such that $i^{*}(x)$ is a generator of $\tilde{h}^{*}(\mathbf{C P}(1))=\tilde{h}^{*}\left(S^{2}\right)$. This element $x$ is then called the Euler class $\chi(L)$ of the canonical line bundle $L$ over $\mathbf{C P}(\infty)$ in the theory $h^{*}\left[1\right.$, p. 37]. With this element $x$ fixed, $h^{*}$ is said to be complex-oriented. 
Some examples of complex-oriented theories are ordinary cohomology, $K$ theory, elliptic cohomology, and complex cobordism. The above definition implies the following properties.

$$
h^{*}\left(\prod_{i=1}^{l}(\mathbf{C P}(\infty))\right) \cong h^{*}(p t)\left[\left[t_{1}, \ldots, t_{l}\right]\right]
$$

$\left(R\left[\left[t_{1}, \ldots, t_{l}\right]\right]\right.$ denotes the graded ring of power series in $l$ variables over a graded ring $R$ ) [1, p. 39].

(B) $h^{*}$ has a formal group law $F$. That is, if $L$ and $M$ are line bundles then

$$
\chi(L \otimes M)=F(\chi(L), \chi(M))
$$

where

$$
F(X, Y)=X+Y+\sum_{i, j \geq 1} a_{i j} X^{i} Y^{j}
$$

$F$ satisfies the obvious commutativity and associativity properties [1, p. 32]. Two examples are the additive and multiplicative group laws $F_{a}$ and $F_{m}$

$$
F_{a}(X, Y)=X+Y, \quad F_{m}(X, Y)=X+Y-u X Y
$$

where $u$ is a unit. $F_{a}$ is the group law for ordinary cohomology and $F_{m}$ is the group law for $K$-theory. If we drop the assumption that $u$ is a unit, these are the only two polynomial group laws over an integral domain [25]. Later, we will give a Lie theoretic criterion for them.

(C) Let $V \rightarrow X$ be a rank $n$ complex vector bundle. Then there is an Euler class $\chi(V) \in h^{2 n}(X)$. Moreover,

$$
\chi(V \oplus W)=\chi(V) \cup \chi(W) .
$$

(D) Thom isomorphism. Let $X^{V}$ be the Thom space of $V$. Since $h^{*}$ is multiplicative, $\tilde{h}^{*}\left(X^{V}\right)$ is an $h^{*}(X)$ module. Then there is a Thom class $U \in$ $\tilde{h}^{2 n}\left(X^{V}\right)$ such that the map

$$
\begin{aligned}
h^{i}(X) & \rightarrow \tilde{h}^{i+2 n}\left(X^{V}\right) \\
x & \mapsto x \cup U
\end{aligned}
$$

is an isomorphism.

(E) Gysin homomorphism. As before, let $\pi: E \rightarrow B$ be a fiber bundle, but require that the fiber $F$ is smooth and almost complex. Then there is a Gysin homomorphism

$$
\pi_{*}: h^{i}(E) \rightarrow h^{i-2 f}(B)
$$

where $2 f$ is the real dimension of $F$ (more generally, the Gysin map is defined for any proper complex-oriented map [23]). $\pi_{*}$ depends on the choice of an orientation and with appropriate choices satisfies the following properties.

(i) $\pi_{*}$ is an $h^{*}(B)$ module map. 
(ii) $(\pi \circ \theta)_{*}=\pi_{*} \circ \theta_{*}$.

(iii) Base change. Consider the following Cartesian square.

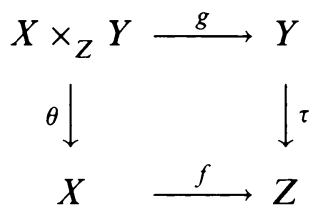

Then $f^{*} \circ \tau_{*}=\theta_{*} \circ g^{*}$.

The Gysin map and the Becker-Gottlieb transfer are related as follows. Let $T_{\pi} \rightarrow E$ be the tangent bundle to the fibers of $\pi$. Assume that the structure group on $F$ preserves the almost complex structure so that $T_{\pi}$ has a complex structure. Then

$$
\tau(\pi)^{*}(x)=\pi_{*}\left(\chi\left(T_{\pi}\right) \cdot x\right)
$$

[6, Theorem 4.3]. Therefore, to obtain a formula for $\pi^{*} \circ \pi_{*}$ it would be helpful to compute $\pi^{*} \circ \tau(\pi)^{*}$. Brumfiel and Madsen give such a formula for the case of a bundle associated to a principal bundle of a compact connected Lie group $G$ with fiber $G / H$ where $H$ is a closed subgroup of maximal rank. More precisely, let $G \supset H \supset T$, where $T$ is a maximal torus of $H$ and therefore of $G$. We have $W_{G}=N_{G}(T) / T$ and $W_{H}=N_{H}(T) / T$ and an inclusion $W_{H} \subset W_{G}$. Let $P \rightarrow B$ be a principal $G$-bundle and let $E_{1}=P \times_{G}(G / T), E_{2}=$ $P \times_{G}(G / H)$, be the associated bundles with fibers $G / T$ and $G / H$. Then we have a commutative diagram

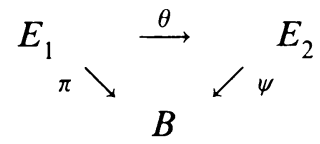

$W_{G}$ and $W_{H}$ act on $E_{1}$ and $E_{2}$ by bundle maps. Therefore a coset $\sigma \in$ $W_{G} / W_{H}$ determines a map

$$
\sigma \circ \theta^{*}: h^{*}\left(E_{1}\right) \leftarrow h^{*}\left(E_{2}\right) .
$$

Theorem 1.3 (Brumfiel-Madsen) [11].

$$
\pi^{*} \circ \tau(\psi)^{*}=\sum_{\sigma \in W_{G} / W_{H}} \sigma \circ \theta^{*}
$$

The proof follows easily from a Mayer-Vietoris property of the transfer [22]. If we let $H=T$, then $\pi=\psi, E_{1}=E_{2}, \theta=\mathrm{id}$, and $W_{H}=1$. Therefore, as a special case of the theorem we obtain the formula

Corollary 1.14 .

$$
\pi^{*} \circ \tau(\pi)^{*}=\sum_{\sigma \in W_{G}} \sigma
$$

Combining 1.2 and 1.4 we have

$$
\pi^{*} \circ \pi_{*}\left(\chi\left(T_{\pi}\right) \cdot x\right)=\sum_{\sigma \in W_{G}} \sigma x
$$


We now specialize to the case where $P=E G$, the universal space for $G$, so $B=B G$ and $E=B T$. For simplicity, we also assume that the coefficients $h^{*}(p t)$ are torsion-free. This assumption is satisfied for the examples discussed in the introduction. See Remark 1.10 for less restrictive assumptions.

The next step is to formally invert $\chi\left(T_{\pi}\right)$ to derive a formula for $\pi^{*} \circ \pi_{*}$. For this procedure to be of any use, we need to prove that $\chi\left(T_{\pi}\right)$ is not a zero-divisor in $h^{*}(B T)$. To establish this fact, we recall some facts from Lie theory.

The fiber of $\pi, G / T$, has a complex structure given as follows. $G$ embeds inside its "complexification" $G_{\mathrm{C}}$, a complex reductive Lie group with maximal compact subgroup $G$. If $B$ is a Borel subgroup of $G_{\mathrm{C}}$ containing $T$, then $G_{\mathbf{C}} / B \cong G / T$. Let $\mathbf{g}$ and $\mathbf{b}$ be the Lie algebras of $G_{\mathbf{C}}$ and $B$. Then with the induced complex structure

$$
T(G / T) \cong G \times{ }_{T} \mathbf{g} / \mathbf{b}
$$

is a complex vector bundle. Hence,

$$
T_{\pi} \cong E G \times{ }_{T} \mathbf{g} / \mathbf{b}
$$

is a complex vector bundle. Let $R^{+}$be a system of positive roots corresponding to $\mathbf{b}$. Then it follows that

$$
T_{\pi} \cong \bigotimes_{\alpha \in R^{+}} L(-\alpha)
$$

where $L(-\alpha)$ is the line bundle over $B T$ induced by the character $e^{-\alpha}$. By the Whitney sum formula,

$$
\chi\left(T_{\pi}\right)=\prod \chi(L(-\alpha)) .
$$

Let $\lambda_{1}, \ldots, \lambda_{l}$ be a system of weights which exponentiate to a generating set for $R(T)$. Then the $L_{i}=L\left(\lambda_{i}\right) \quad(i=1, \ldots, l)$ generate the line bundles over $B T$. Complex orientability implies that $\chi\left(L_{i}\right)$ are not zero-divisors. We have

$$
L(-\alpha) \cong \bigotimes L_{i}^{m_{i}}, \quad m_{i} \in \mathbf{Z}, \text { not all } m_{i}=0 .
$$

By our discussion of formal group laws,

$$
\chi(L(-\alpha))=\sum m_{i} \chi\left(l_{i}\right)+\text { higher order terms } .
$$

Hence we have

Proposition 1.7. If $h^{*}$ is torsion-free, $\chi(L-(\alpha))$ is not a zero-divisor.

Proof. This is an easy argument using the graded structure of $h^{*}(B T)$.

Theorem 1.8. If $h^{*}$ is complex-oriented, then for $x \in h^{*}(B T)$,

$$
\pi^{*} \circ \pi_{*}(x)=\sum_{\sigma \in W} \sigma \frac{1}{\prod \chi(L(-\alpha))}(x) .
$$

Proof. By construction

$$
\pi^{*} \circ \pi_{*}: h^{*}(B T) \rightarrow h^{*}(B T)
$$


is an $h^{*}(B G)$-module homomorphism, as is $\pi^{*} \circ \tau(\pi)^{*}$. Since rationally $h^{*}(B G)$ $=h^{*}(B T)^{W}$, and there is no torsion in $h^{*}(B T)$, these are also $h^{*}(B T)^{W}$. module homomorphisms. Let

$$
S=\prod_{\sigma \in W} \sigma \chi\left(T_{\pi}\right) \in h^{*}(B T)
$$

and let $Q=h^{*}(B T)\left[S^{-1}\right]$. By standard results from commutative algebra, there is a unique extension $\phi$ which makes the following diagram commute.

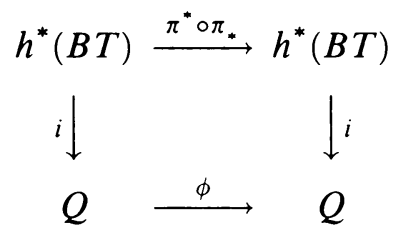

Similarly, we have an extension $\psi$ and a commutative diagram

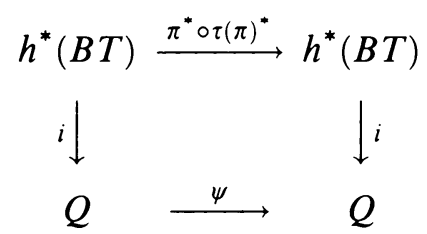

and $\psi(x)=\phi\left(\chi\left(T_{\pi}\right) \cdot x\right)$. By 1.6 and 1.7, $\chi\left(T_{\pi}\right)$ is not a zero-divisor. Also, $\sigma \chi\left(T_{\pi}\right)$ is not a zero-divisor for $\sigma \in W$, so $S$ is not a zero-divisor. Hence, the vertical arrows in the above diagrams are inclusions. Moreover, in $Q$, multiplication by $\chi\left(T_{\pi}\right)$ is an isomorphism, so we can invert it to give $\psi\left(1 / \chi\left(T_{\pi}\right) \cdot x\right)=$ $\phi(x)$. From 1.5 it follows that for $y \in Q$,

$$
\psi(y)=\sum_{\sigma \in W} \sigma y .
$$

Hence, for $x \in h^{*}(B T)$,

$$
\pi^{*} \circ \pi_{*}(x)=\sum_{\sigma \in W} \sigma \frac{1}{\chi\left(T_{\pi}\right)} x=\sum_{\sigma \in W} \sigma \frac{1}{\Pi \chi(L(-\alpha))} x .
$$

Corollary-Definition 1.9. Let $G=H_{i}$, a rank one compact Lie group and let $\pi=\pi_{i}$. Let $\alpha_{i}$ and $s_{i}$ be the positive root and nontrivial element of the Weyl group of $H_{i}$. Then if $h^{*}$ is torsion-free

$$
D_{i}=\pi_{i}^{*} \circ \pi_{i *}=\left(1+s_{i}\right) \frac{1}{\chi(L(-\alpha))} .
$$

Remark 1.10. We assumed that our coefficients $h^{*}(p t)$ are torsion-free. In fact, it suffices to assume that they are free of two-torsion. To extend the result to this case, one needs to prove that $\chi\left(L\left(-\alpha_{i}\right)\right)$ is not a zero-divisor. This fact follows from the fact that at least one of the integers $m_{i}$ relating roots to weights has absolute value less than or equal to two. More generally, if $G$ is of adjoint type we need no assumptions on torsion. For more details see [14]. 
Remark 1.11. It is easy to see that these operators $D_{i}$ are related to corresponding operators defined on $h^{*}(G / T)$. Indeed, $G \rightarrow G / T$ is a principal $T$-bundle so it is induced by a classifying map $\theta: G / T \rightarrow B T$. Therefore, we have the "characteristic homomorphism"

$$
\theta^{*}: h^{*}(B T) \rightarrow h^{*}(G / T) .
$$

Similarly, for rank one subgroups $H_{i}$ of $G$ corresponding to simple roots we have maps $\theta_{i}: G / H_{i} \rightarrow B H_{i}$. Moreover,

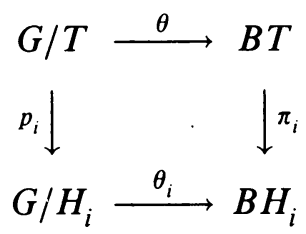

is a pull-back diagram. Base change implies that $p_{i}^{*} \circ p_{i *} \circ \theta^{*}=\theta^{*} \circ D_{i}$. Hence, $\theta^{*}$ intertwines the actions of $D_{i}$ and $p_{i}^{*} \circ p_{i *}$, which we denote $C_{i}$. Moreover, after tensoring with the rationals, the map $\theta^{*}: h^{*}(B T) \rightarrow h^{*}(G / T)$ is surjective, as is easily seen by using the naturality of the Atiyah-Hirzebruch spectral sequence and the fact that $\theta^{*}$ is surjective for the case of rational cohomology. Therefore, to compute the operators $C_{i}$ it suffices to compute the operators $D_{i}$.

\section{APPLICATIONS TO ORDINARY COHOMOLOGY AND $K$-THEORY}

In this section we interpret Theorem 1.8 in two familiar settings to obtain some classical results. First, let $h^{*}$ be $H^{*}$, ordinary cohomology with complex coefficients. Then

$$
H^{*}(B T) \cong C\left[\left[\lambda_{1}, \ldots, \lambda_{l}\right]\right]
$$

under the identification $\chi(L(\lambda))=\lambda$. Given this identification,

$$
\pi^{*} \circ \pi_{*}(x)=\sum_{\sigma \in W} \sigma \frac{1}{\Pi_{\alpha \in R^{+}}-\alpha}(x) .
$$

When $G=H_{i}$ is rank one, this composition is just the classical BGG operator

$$
A_{i}=\frac{1}{\alpha_{i}}\left(s_{i}-1\right) \text {. }
$$

These formulas have previously been obtained in [2] using zero sets of holomorphic vector fields.

Applying Theorem 1.8 to $K$-theory gives somewhat more interesting results. We map the representation ring $R(T)$ to $K(B T)$ by mapping $e^{\lambda}$ to $[L(\lambda)]$, the class of the line bundle defined by $\lambda$. In $K$-theory we take $\chi(L)=[1]-[L]$, where [1] is the class of the trivial line bundle. With these conventions, when we apply 1.8 to $e^{\lambda}$, we obtain

$$
\pi^{*} \circ \pi_{*}([L(\lambda)])=\sum_{\sigma \in W} \sigma \frac{1}{\prod_{\alpha \in R^{+}}\left(1-e^{-\alpha}\right)} e^{\lambda}
$$


Let $\rho=\frac{1}{2} \sum_{\alpha \in R^{+}} \alpha$. Then this formula easily reduces to

$$
\pi^{*} \circ \pi_{*}([L(\lambda)])=\frac{1}{\prod_{\alpha \in R^{+}}\left(e^{\alpha / 2}-e^{-\alpha / 2}\right)} \sum_{\sigma \in W}(-1)^{l(\sigma)} e^{\sigma(\lambda+\rho)}
$$

which is the classical form of the Weyl character formula.

Theorem 2.5 (Weyl). The character of the irreducible representation of highest weight $\lambda$ is given in (2.4).

Proof. We need to show that $\pi^{*} \circ \pi_{*}([L(\lambda)])$ is the vector bundle corresponding to the character of the irreducible representation of highest weight $\lambda$. By the Grothendieck version of the Riemann-Roch theorem [24] extended to classifying spaces the Gysin map $K(B T) \rightarrow K(B G)$ is the same as

$$
\pi_{!}: K(B T) \rightarrow K(B G)
$$

where $\pi_{1}(L(\lambda))$ is the virtual vector bundle corresponding to

$$
\sum(-1)^{i} H^{i}\left(G_{\mathbf{C}} / B, L(\lambda)\right)
$$

$H^{i}(X, L)$ refers to the $i$ th anti-holomorphic Čech cohomology group. The Borel-Weil-Bott theorem asserts that $H^{i}\left(G_{\mathrm{C}} / B, L(\lambda)\right)=0$ for $i>0$, and $H^{0}\left(G_{\mathbf{C}} / B, L(\lambda)\right)$ is the irreducible representation of highest weight $\lambda$.

Remark 2.7. This argument is a mild variation on the proof of the Weyl character formula obtained by Atiyah and Bott [4]. This variation replaces their generalized Lefschetz fixed-point formula with the Riemann-Roch theorem and some formal calculations in homotopy theory.

In the case where $G=H_{i}$ is rank one, the operator $\pi_{i}^{*} \circ \pi_{i *}$ is the Demazure operator $B_{i}$. It has the form

$$
B_{i}=\frac{1}{1-e^{-\alpha_{i}}}\left(1-e^{-\alpha_{i}} s_{i}\right) .
$$

Thus, we can recover the standard formulas for the Demazure operators as well as for the BGG operators.

It is well known that the expression

$$
M=\frac{1}{\prod_{\alpha \in R^{+}}\left(1-e^{-\alpha}\right)} e^{\lambda} \in K(B T)\left[\frac{1}{\chi\left(T_{\pi}\right)}\right]
$$

which appeared in (2.3) is important in representation theory. It is the formal character of the Verma module of highest weight $\lambda$ associated to the Lie algebra g of $G[16$, p. 130]. A construction related to the transfer gives a topological interpretation of this expression. The bundle $T_{\pi} \rightarrow B T$ has a complementary bundle $\beta$ such that $T_{\pi} \oplus \beta=[N]$, the rank $N$ trivial bundle. Let $\sigma$ and $\Phi$ be the Thom isomorphisms to the Thom spaces of $[N]$ and $\beta$ respectively. Then 
we have the following commutative diagram,

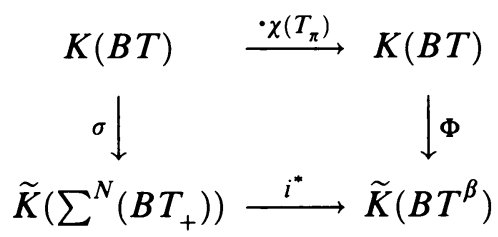

Then taking $M$ in $K(B T)\left[\frac{1}{\chi\left(T_{\pi}\right)}\right]$, the composition

$$
M \rightarrow M \cdot \chi\left(T_{\pi}\right) \rightarrow \Phi\left(e^{\lambda}\right)
$$

maps $M$ to an element of $K\left(B T^{\beta}\right)$. On the other hand, $i^{*} \circ \sigma$ is equivariant with respect to the Weyl group action on $B T$ because $\sigma$ is the suspension isomorphism. Moreover, all these maps are inclusions. Hence we can regard $M$ as an element of $K\left(B T^{\beta}\right)$. Thus, this procedure gives a topological construction of a well-known object in representation theory. It is easy to check that Weyl group orbits of $M$ correspond to Verma modules with a given central character. Moreover, one can use a modification of the transfer used in 1.6 to give a topological construction of the irreducible quotients of Verma modules associated to Weyl group elements with smooth Schubert varieties. It would be interesting to try to develop these constructions further. A full account of this description of Verma modules appears in [14].

\section{BRAid RELATIONS}

In this section, we will be concerned with the relations which the operators $D_{i}$ satisfy.

\section{Proposition 3.1.}

$$
D_{i}^{2}=D_{i}(1) D_{i}=\left[\frac{1}{\chi\left(L\left(-\alpha_{i}\right)\right)}+\frac{1}{\chi\left(L\left(\alpha_{i}\right)\right)}\right] D_{i} .
$$

Proof. This follows from the projection formula

$$
\pi_{i *}\left(\pi_{i}^{*}(y) \cdot 1\right)=y \cdot \pi_{i *}(1) .
$$

Remark 3.2. In ordinary cohomology $\chi\left(L\left(-\alpha_{i}\right)\right)=-\chi\left(L\left(\alpha_{i}\right)\right)$ so $D_{i}^{2}=0$. In $K$-theory the formal group law is

$$
F(X, Y)=X+Y-X Y,
$$

which implies $D_{i}^{2}=D_{i}$. We have

Proposition 3.3. $W$ acts faithfully on $h^{*}(B T)$.

Proof. It is a consequence of definitions that the Atiyah-Hirzebruch spectral sequence degenerates for $h^{*}(B T)$. Hence, $h^{*}(B T) \cong H^{*}(B T, \mathbf{Z}) \widehat{\otimes} h^{*}(p t)$ as graded $h^{*}(p t)$ modules. This isomorphism is $W$-equivariant on the associated graded level. The result follows from the fact that $W$ acts faithfully on $H^{*}(B T, \mathbf{Z})$. 
Corollary 3.4. If $h^{*}$ is torsion-free, the elements $w \in W$ are linearly independent over $h^{*}(B T)$ as operators on $h^{*}(B T)$.

Proof. Suppose first that $h^{*}$ is a domain. It is a well-known result of Artin that if a finite group acts faithfully on a field, then the group elements are linearly independent over the field [3, p. 35]. We may apply Artin's result to the fraction field of the domain $h^{*}(B T)$. The general case follows from a refinement of Artin's result (see Remark 3.13 or $[14,13.7]$ ).

We are interested in determining when these operators $D_{i}$ satisfy braid relations, which are defined as follows. The Weyl group is a free group on $l$ generators $s_{1}, \ldots, s_{l}$ modulo certain relations. These relations fall into two classes. First, one requires that $s_{i}^{2}=1$, and we will largely ignore these relations. Second, one requires that there exist integers $m_{i j}$ such that $s_{i} s_{j} s_{i} \cdots=s_{j} s_{i} s_{j} \cdots$ (where there are $m_{i j}$ terms on each side). These relations are called braid relations since the special case when $W$ is the symmetric group on $n$ letters they are the defining relations for Artin's braid group. Since the Weyl group is a finite group, $m_{i j}$ is always finite and in fact equal to $2,3,4$, or 6 . We can now give

Definition 3.5. The operators $D_{i}$ satisfy braid relations if $D_{i} D_{j} D_{i} \cdots=$ $D_{j} D_{i} D_{j} \cdots$, where there are $m_{i j}$ terms on each side.

Remark 3.6. If the operators $D_{i}$ satisfy braid relations, we can define an operator $D_{w}$ for $w \in W$ as follows. We take a minimal decomposition of $w=s_{i_{1}} \cdots s_{i_{k}}$ and then we set $D_{w}=D_{i_{1}} \cdots D_{i_{k}}$. A theorem of Matsumoto $\left[9\right.$, p. 16] implies that $D_{w}$ does not depend on the choice of a minimal decomposition.

We can now state and prove our main theorem.

Theorem 3.7. Let $G$ be a compact connected Lie group with at least two nonorthogonal roots and let $h^{*}$ be a complex-oriented cohomology theory with $h^{*}$ torsion-free. Let $D_{i}(i=1, \ldots, l)$ be the operators defined above. Then the operators $D_{i}$ satisfy braid relations if and only if the formal group law is polynomial.

Remark 3.8. Of course, there are no braid relations to check unless there are at least two nonorthogonal roots. This condition only implies that there is a simple factor of rank at least 2 .

Proof of 3.7. There are three cases to consider. These cases are when the two nonorthogonal simple roots $\alpha_{i}$ and $\alpha_{j}$ have $m_{i j}=3$, 4, or $6\left(m_{i j}=2\right.$ implies the roots are orthogonal). We do the case $m_{i j}=3$ explicitly and refer the reader to Gutkin's extension of our result for the remaining two cases.

We need to evaluate the identity

$$
D_{1} D_{2} D_{1}=D_{2} D_{1} D_{2}
$$

Here $D_{i}=\left(1+s_{i}\right) \frac{1}{\chi\left(L\left(-\alpha_{i}\right)\right)}$. By (3.4), the operators $w \in W$ are linearly independent over $Q$. Hence we can check (3.9) by expanding each side and equating 
coefficients. We will write $g(\alpha)$ for $\chi(L(-\alpha))$ for convenience. When we examine terms with coefficient $s_{1}$ we get

$$
\begin{gathered}
\left(\frac{1}{g\left(-\alpha_{1}\right)} \frac{1}{g\left(\alpha_{1}+\alpha_{2}\right)} \frac{1}{g\left(\alpha_{1}\right)}+\frac{1}{g\left(\alpha_{1}\right)} \frac{1}{g\left(\alpha_{2}\right)} \frac{1}{g\left(-\alpha_{1}\right)}\right) s_{1} \\
=\frac{1}{g\left(\alpha_{2}\right)} \frac{1}{g\left(-\alpha_{1}\right)} \frac{1}{g\left(\alpha_{1}+\alpha_{2}\right)} s_{1}
\end{gathered}
$$

since $s_{1}\left(\alpha_{2}\right)=\alpha_{1}+\alpha_{2}$ and $s_{1}\left(\alpha_{1}\right)=-\alpha_{1}$. After clearing denominators, we get

$$
g\left(\alpha_{1}\right) g\left(\alpha_{2}\right)+g\left(-\alpha_{1}\right) g\left(\alpha_{1}+\alpha_{2}\right)=g\left(\alpha_{1}\right) g\left(-\alpha_{1}\right) .
$$

It is easy to see that

$$
g\left(-\alpha_{1}\right)=\sum_{k \geq 1} b_{k} g\left(\alpha_{1}\right)^{k}
$$

with $b_{1}=-1\left[1\right.$, p. 45]. Replace $g\left(\alpha_{1}\right)$ by $X$ and $g\left(\alpha_{2}\right)$ by $Y$. Then by using the formal group law, we get

$$
X Y=-\left(\sum_{k \geq 1} b_{k} X^{k}\right)\left(Y+\sum_{k, l \geq 1} a_{k l} X^{k} Y^{l}\right) .
$$

Since the left-hand side has no expressions with powers of $Y$ greater than 1 , neither does the right-hand side. The expression

$$
g\left(-\alpha_{1}\right)=\sum_{k \geq 1} b_{k} X^{k}
$$

is not a zero-divisor so $a_{k l}=0$ for $l>1$. But $a_{k l}=a_{l k}$, so the formal group law has the form

$$
F(X, Y)=X+Y+a_{11} X Y .
$$

Thus, $F$ is polynomial and if $a_{11}=0$ or is a unit, then $F$ is the formal group law of cohomology or of $K$-theory.

For the cases $m_{i j}=4$ and 6 a similar argument works. However, the calculations are burdensome and we will not present them here. Instead, we explain how our earlier paper combined with Gutkin's work solves the problem. Since $h^{*}$ is torsion-free, we can replace $\chi\left(L\left(\alpha_{i}\right)\right)$ by a formal power series $\exp \left(\alpha_{i}\right)$ in $L\left(\alpha_{i}\right)$ called the exponential. exp has the property that $\exp (L \otimes$ $M)=F(\exp (L), \exp (M))$. Now Gutkin [15] has established necessary and sufficient conditions that an operator of the form

$$
D_{i}=\left(1 / f\left(\alpha_{i}\right)+s_{i} 1 / g\left(\alpha_{i}\right)\right)
$$

satisfy braid relations for the cases $m_{i j}=4$ or 6 (the authors [10] proved the result for $\left.m_{i j}=3\right)$. In particular, taking $f=g$, braid relations imply that $g\left(\alpha_{i}\right)=c \alpha_{i}$ or $c\left(e^{a \alpha_{i}}-1\right)$, from which one sees easily that the cohomology theory in question satisfies the formal group law of either cohomology or $K$ theory. 
Remark 3.12. One sees trivially that Theorem 3.7 extends to affine Kač-Moody groups. In the Kač-Moody case, the groups corresponding to $T$ and $H_{i}$ are still finite dimensional compact Lie groups so we can use the transfer to define the operators in the same way.

Remark 3.13. We assumied that our coefficients $h^{*}(p t)$ are torsion-free. In fact, it suffices that they are free of 2-torsion. To extend the result to this case, one needs to prove that the Weyl group elements are linearly independent over $h^{*}(B T)$. The linear independence of Weyl group elements follows from two facts. First, the Weyl group acts faithfully on $H^{*}(B T, Z / p)$ for $p \neq 2$. Second, the argument Artin gives for linear independence [3, p. 35] implies that if for all pairs $w_{1}$ and $w_{2} \in W$, there exists $x \in h^{*}(B T)$ such that $w_{1}(x)-w_{2}(x)$ is not a zero-divisor, then Weyl group elements are independent over $h^{*}(B T)$. At least in the rank two situation (the only situation we need to consider), there exist line bundles whose Euler classes satisfy this property.

\section{REFERENCES}

1. J. F. Adams, Stable homotopy and generalised homology, Univ. of Chicago Press, 1974.

2. E. Akyildiz and J. B. Carrell, Zeros of holomorphic vector fields and the Gysin homomorphism, Proc. Sympos. Pure Math., vol. 40, Amer. Math. Soc., Providence, R.I., 1983, pp. 47-54.

3. E. Artin, Galois theory, Notre Dame lecture notes, 1959.

4. M. F. Atiyah and R. Bott, A Lefschetz fixed point theorem for elliptic complexes. II, Ann. of Math. 88 (1968), 451-491.

5. M. F. Atiyah and F. Hirzebruch, Vector bundles and homogeneous spaces, Proc. Sympos. Pure Math., vol. 3, Amer. Math. Soc., Providence, R.I., 1961, pp. 7-38.

6. J. C. Becker and D. H. Gottlieb, The transfer map and fiber bundles, Topology 14 (1975), $1-12$.

7. J. N. Bernstein, I. M. Gel' fand, S. I. Gel'fand, Schubert cells and the cohomology of spaces $G / P$, Russian Math Surveys 28 (1973), 1-26.

8. A. Borel and F. Hirzebruch, Characteristic classes and homogeneous spaces. I, Amer. J. Math. 80 (1958), 458-538.

9. N. Bourbaki, Groupes et algèbres de Lie, Hermann, Paris, 1968, Chapitres 4, 5, et 6.

10. P. Bressler and S. Evens, On certain Hecke rings, Proc. Nat. Acad. Sci. U.S.A. 84 (1987), 624-625.

11. G. Brumfiel and I. Madsen, Evaluation of the transfer and universal surgery classes, Invent. Math. 32 (1976), 133-169.

12. M. Demazure, Désingularisation des variétés de Schubert, Ann. Ecole Norm. Sup. (4) 7 (1974), 53-88.

13. E. Dyer, Cohomology theories, Benjamin, 1969.

14. S. Evens, The transfer for compact Lie groups, induced representations, and braid relations, Thesis MIT, 1988.

15. E. Gutkin, Representations of Hecke algebras, Trans. Amer. Math. Soc. 307, 1988.

16. J. E. Humphreys, Introduction to Lie algebras and representation theory, Springer-Verlag, 1972.

17. V. Kač, Constructing groups associated to infinite dimensional Lie algebras, Infinite Dimensional Groups with Applications, Springer-Verlag, 1985, pp. 167-217.

18. D. Kazhdan and G. Lusztig, Equivariant $K$-theory and representation of Hecke algebras. II, Invent. Math. 80 (1985), 209-231. 
19. B. Kostant and S. Kumar, The nil Hecke ring and cohomology of G/P for a Kač-Moody group $G$, Adv. in Math. 62 (1986), 187-237.

20. B. Kostant and S. Kumar, T-equivariant $K$-theory of generalized flag varieties, Proc. Nat. Acad. Sci. U.S.A. 84 (1987), 4351-4354.

21. G. Lusztig, Equivariant $K$-theory and representations of Hecke algebras, Proc. Amer. Math. Soc. 94 (1985), 337-342.

22. S. A. Mitchell and S. B. Priddy, A double coset formula for Levi subgroups and splitting $B G L_{n}$, preprint, 1987.

23. D. G. Quillen, Elementary proofs of some results of cobordism theory using Steenrod operations, Adv. in Math. 7 (1971), 29-56.

24. M. F. Atiyah and F. Hirzebruch, The Riemann-Roch theorem for analytic embeddings, Topology 1 (1962), 151-166.

25. M. Hazewinkel, Formal groups and applications, Academic Press, 1978.

Department of Mathematics, Massachusetts Institute of Technology, Cambridge, MASSACHUSETTS 02139

Current address (Paul Bressler): Department of Mathematics, Purdue University, West Lafayette, Indiana 47907

Current address (Sam Evens): Department of Mathematics, Rutgers University, New Brunswick, New Jersey 08903 